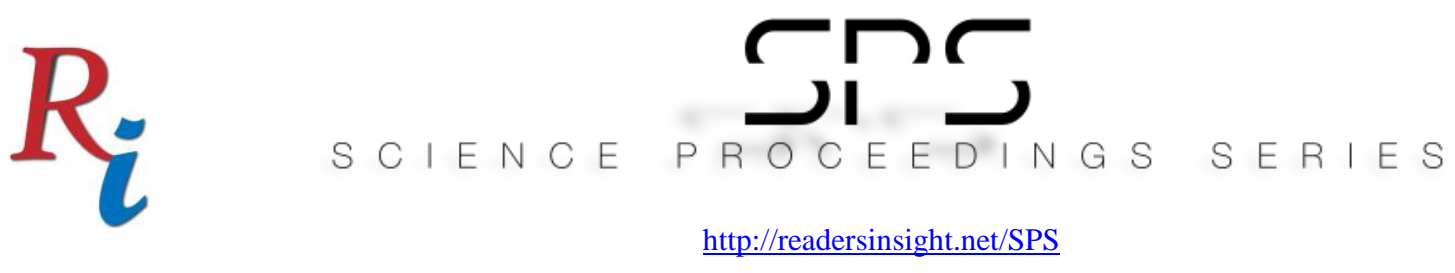

\title{
UWB WITH AGV FOR LOCALIZATION SYSTEM
}

\author{
Hameedah Sahib Hasan \\ Division of Control and Mechatronics Engineering, School of Electrical Engineering \\ Faculty of Engineering, UTM, Johor \\ Malaysia. \\ Ministry of Higher Education and Scientific Research \\ Iraq \\ hameedah211ou@gmail.com
}

\section{Mohamad Shukri Zainal Abidin*}

Division of Control and Mechatronics Engineering, School of Electrical Engineering, Faculty of Engineering, UTM, Johor,

Malaysia.

shukri@utm.my

\section{Mohd Saiful Azimi Mahmud}

Division of Control and Mechatronics Engineering

School of Electrical Engineering, Faculty of Engineering

UTM, Johor

Malaysia.

*Corrosponding author's Email: shukri@utm.my

Peer-review under responsibility of $5 t^{d}$ Asia International Conference 2020 Scientific Committee http://connectingasia.org/scientific-committee/

(C) 2020 Published by Readers Insight Publisher, lat 306 Savoy Residencia, Block 3 F11/1,44000 Islamabad. Pakistan,

editor@ readersinsight.net

This is an open access article under the CC BY-NC-ND license (http://creativecommons.org/licenses/by-nc-nd/4.0/). 

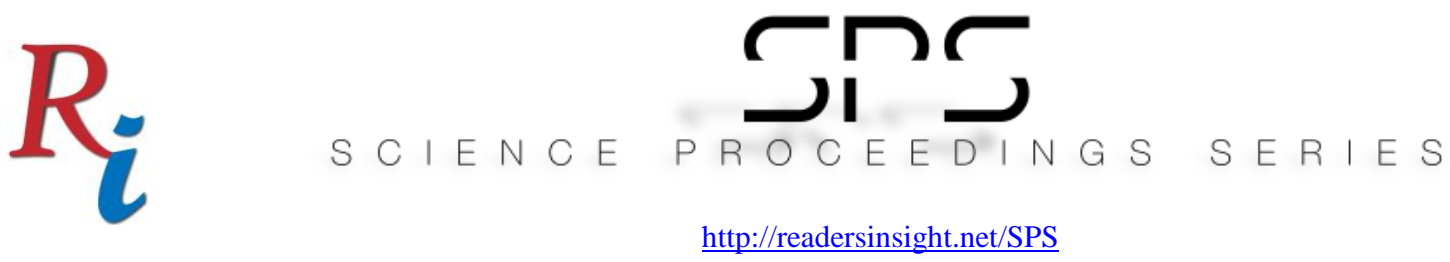

\section{A b s tra c t}

Automated guided vehicle (AGV) has great significance in material flow and distribution of goods with high precision and efficiency. Besides, Ultra-wideband (UWB) is a technology used to transmit wireless signal in nanosecond. In this paper, localization system using AGV via UWB is designed. Futhermore, the experiment of localization with AGV is introduced and the result is disscused. In addition, control system for AGV is developed by using kinematic control system via Labview.

Keywords - UWB Technology; Localization; AGV; Kinematic Control.

\section{Rese a r ch H i g h I ight s}

AGV has played an important role to improve the efficiency of the enterprise especially in transportation. It takes the task from starting node to a destination node in the given storage environment(1). In term of localization, LPS has several technologies to get position such as Wi-Fi, Bluetooth, RFID, IR, Ultrasonic and Ultra-wideband (UWB). UWB is a wireless technology used ultra -short pulse based on radio signals. It has many features and has been implemented in many LPS application(2). The aims of this paper are to discuss localization system using UWB technology as well as the experiment of localization with AGV.

\section{UWB technology:}

The UWB signal is a short-rang radio type device that uses ultra-short pulse and this reaches within nanosecond to calculate a position. UWB has many features in term of transmission and it has high data rate with low power transmission. At the same time, it has large bandwidth with low power consumption, high multipath immunity (3) and high resolution. All these features led UWB to become an important technology in various applications in LPS such as position tracking. For positioning system, GPS has been widely used since 1995, where 24 satellites were used to obtain full functional GPS. However, GPS has some constraints in specific area where it is unable to transmit GPS signal inside the building due to insufficient amount of line of sight ( $\mathrm{LoS})$. Those constrains causes to signal to be blocked thus multipath signals will be produced which lead to the change of signal direction and finally affect the accuracy of GPS data (4)(5). Therefore, UWB is selected to be implemented in this paper due to its abilities to overcome those constrains in localization system.

Table 1. UWB Features

\begin{tabular}{|l|l|}
\hline Size & Dimension :29mm x 52.7mm \\
\hline Power Consumption & Universal PCB, can be flashed to operate as an anchor or as a tag. \\
\hline
\end{tabular}



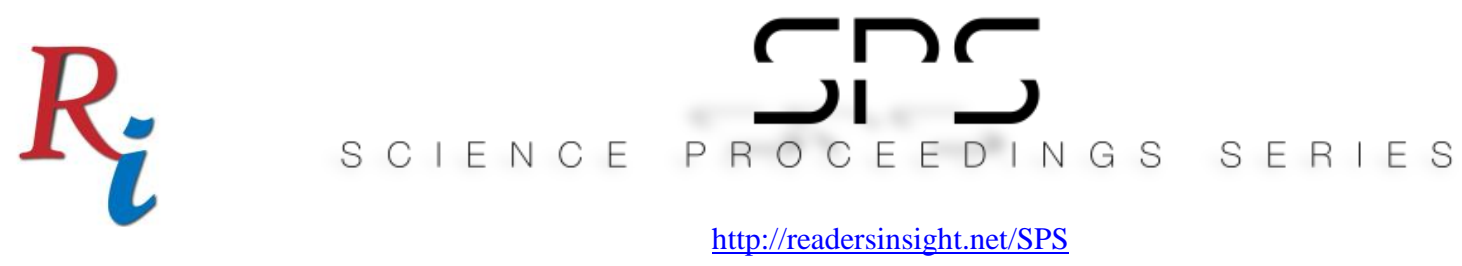

\begin{tabular}{|l|l|}
\hline $\begin{array}{l}\text { DWM1000 } \\
\text { Transceiver }\end{array}$ & UWB Decawave Localization Transceiver (DWM1000 \\
\hline
\end{tabular}

\section{Results}

Figure 1 shows the kinematic control for AGV motion where the target point $(x d, y d)$ is selected as $(2,2)$. The kinmatic parameters that used is $k y, l y, l x, k x$ which selected $=1$ and the real motion for AGV shows, it can reach point $(2,1.99)$.

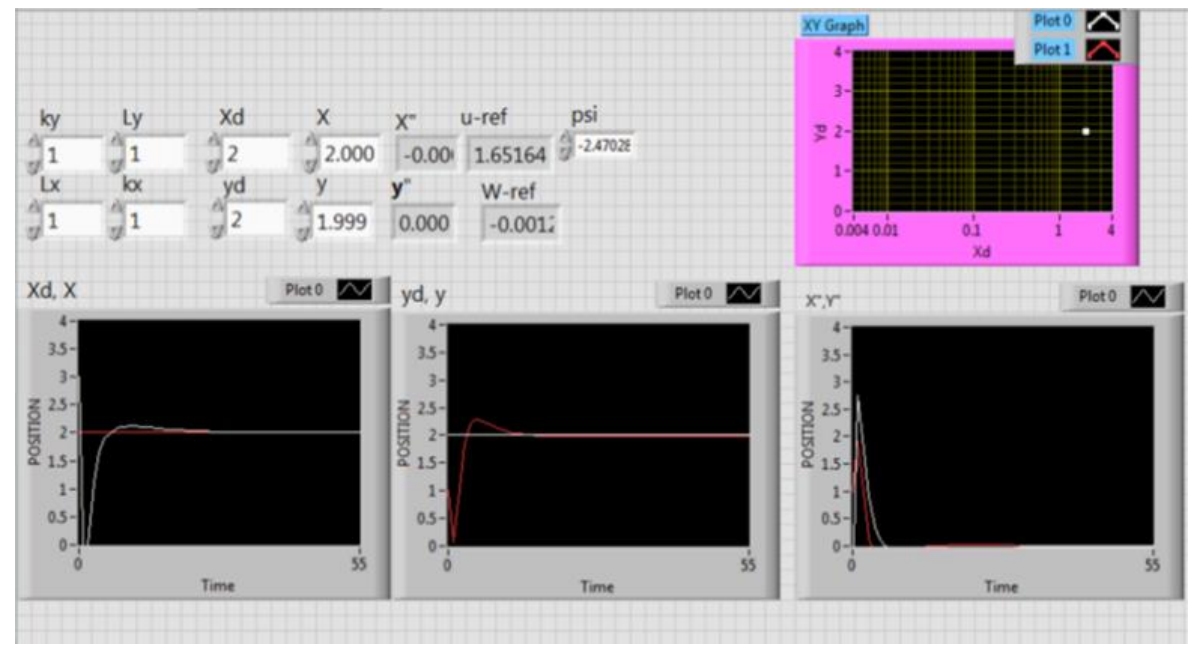

Figure 1. Kinematic Control for Point $(2,2)$

In Figure 2, the AGV motion is started from point $(0.48,0.33)$. The AGV moved smoothly till it reached to the desired position at node $(3,2.5)$ that is selected inside the front panel. The real position that $\mathrm{AGV}$ reached is $(3.05,2.51)$ for $(x, y)$ position. The error in $x$ - axis is $0.05 \mathrm{~m}$ and in $\mathrm{y}$ - axis is $0.01 \mathrm{~m}$. At the same time, the AGV motion in real time is very near to the required destination shown in Figure 2.

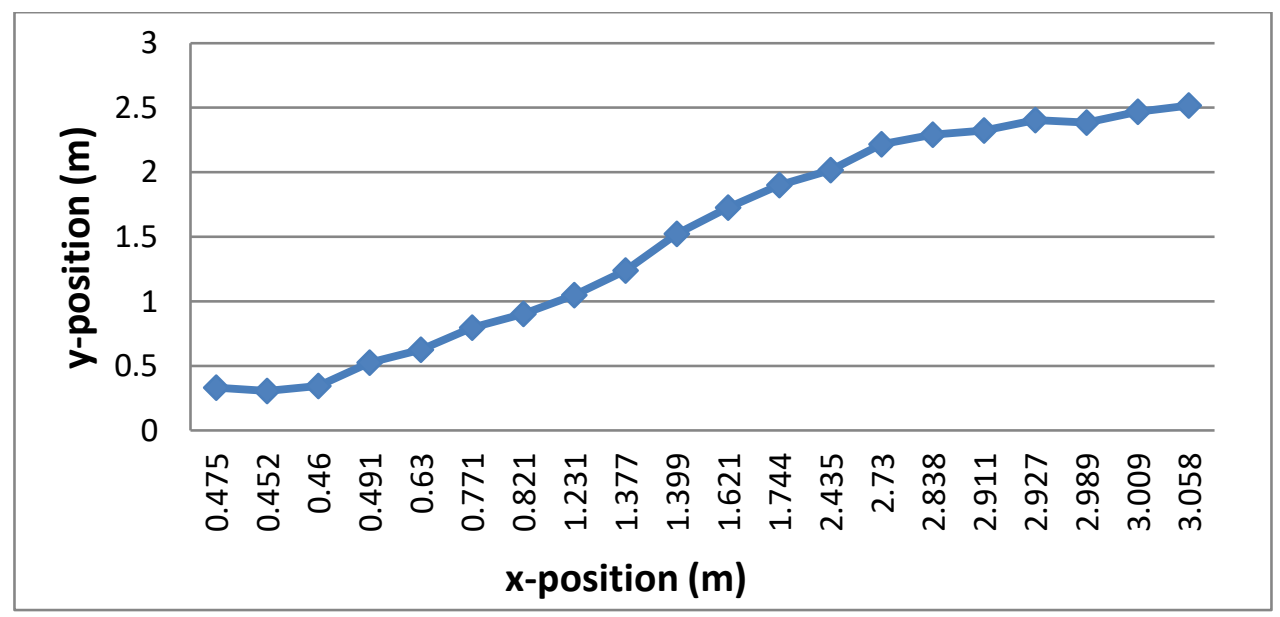

Figure 2. Robot Motion (3, 2.5)

Copyright (C) 2020 Authors. This is an open access article distributed under the Creative Commons Attribution License, which permits unrestricted use, distribution, and reproduction in any medium, provided the original work is properly cited. 


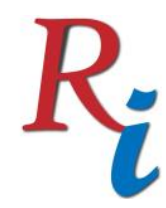

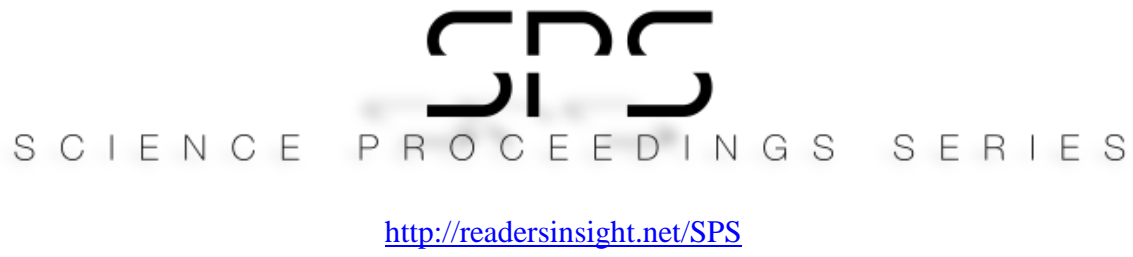

http://readersinsight.net/SPS

\section{Conclusion}

In this paper, the experiment of AGV localization system is discused. In addition, the results for AGV motion are also discussed. Furthermore, the experiment resuts has shown that the $\mathrm{AGV}$ is able to navigate smoothly to reach the target location.

\section{Acknowledgement}

The authors are grateful to the Universiti Teknologi Malaysia and the Ministry of Education (MOE), for their partial financial support through their research funds, Vote No. Q.J130000.2651.17J53.

\section{REFERENCES}

[1] Qing G, Zheng Z, Yue X. Path-planning of Automated Guided Vehicle based on Improved Dijkstra Algorithm. 2017 29th Chinese Control Decis Conf. 2017;7138-43.

[2] Hameedah Sahib Hasan, M.Shukri Zainal Abidin, MSA Mahmud MFMS. Automated guided vehicle routing: Static, dynamic and free range. Int J Eng Adv Technol. 2019;8(5C):1-7.

[3] Zhao L, Psota ET, Perez LC. A comparison between UWB and TDOA systems for smart space localization. IEEE Int Conf Electro Inf Technol. 2014;179-83.

[4] Hu S, Kang M, She C. Vehicle positioning based on UWB technology. J Phys Conf Ser [Internet]. 2017;887:012069. Available from: http://stacks.iop.org/17426596/887/i=1/a=012069?key=crossref.922d0a068c23be1 af66976c53e9a3633

[5] Hameedah Sahib Hasan, Mohamed Hussein SMS and MAMD. An Overview of Local Positioning System : Technologies, Techniques and Applications. Int J Eng Technol. 2018;7:1-5. 